\title{
03 | Tecnología posible. El mobiliario como vehículo de modernización de la arquitectura española de los años $\mathbf{3 0}$ Possible technology. The furniture as a vehicle for the modernization of Spanish architecture in the $30^{5} \_$Maria Villanueva Fernández, Héctor
}

\section{García-Diego Villarías}

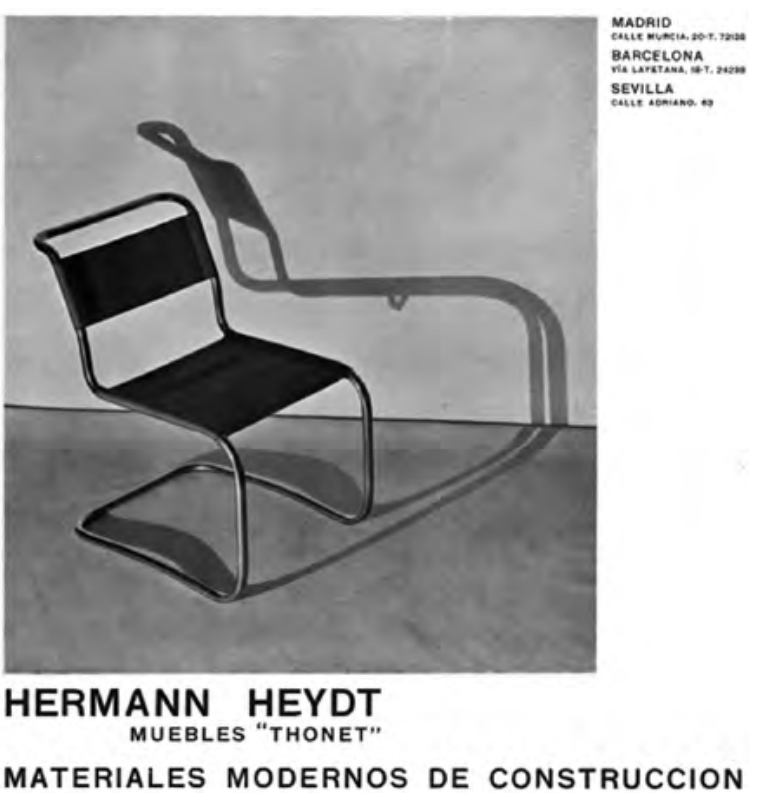

[1]

\section{El Arquitecto y las empresas de mobiliario}

A lo largo de la historia el arquitecto ha cobrado especial protagonismo en el campo del diseño de mobiliario, ya fuese por el desarrollo vocacional de la creatividad o bien por la mera necesidad de amueblar sus obras. Fue a partir de las últimas décadas del siglo XIX, cuando su labor en materia del mueble se intensificó. Si bien al inicio de este periodo el arquitecto desarrolló proyectos integrales en los que la arquitectura y el mobiliario -así como el resto de objetos decorativos- constituyeron las partes de un todo denominado "obra de arte total" 1 , en las primeras décadas de siglo XX emergió como creador de tipos únicos, proyectados como objetos acabados con una forma, aparentemente derivada de una función concreta aunque, en ocasiones, ideados para una arquitectura específica.

La transformación técnica originada a comienzos del siglo $X X$ en todas las áreas $y$, en especial, en las artes visuales y en la arquitectura, requirió una profunda modificación del concepto de mobiliario, alejada de reformas superficiales formales, propias de los estilos, como había sucedido hasta entonces ${ }^{2}$. La revolución industrial exigía otra formal que no se produjo hasta décadas después. Por un lado, la industria propuso técnicas de producción ligadas al progreso y, por otro, los diseñadores ofrecieron productos decorados, vinculados a las formas de la artesanía y de los estilos de la época. De manera que, los diseñadores siguieron creando el mismo tipo de moblaje pero industrialmente, de una forma repetitiva y mecanizada, siendo estas formas inadecuadas para esos métodos de producción, pero apropiadas para el desarrollo tecnológico. Esta situación provocó que industria y diseño se desarrollaran a dos velocidades distintas.

Tras la I Guerra Mundial se produjo un progreso sustancial de la tecnología que fue utilizado en la arquitectura como elemento diferenciador de modernidad. Aquel progreso tecnológico no solo constituyó un avance en la experimentación material y de producción, sino que también proporcionó una novedosa estética moderna, que cristalizó, de manera destacada y con una mayor velocidad, en el mobiliario que equipaba la nueva arquitectura. Mientras que las innovaciones tecnológicas penetraban de manera gradual, no sin esfuerzo, en los procesos constructivos de la arquitectura, el mobiliario constituía un terreno abonado para la experimentación y puesta a prueba de las posibilidades de las nuevas técnicas de manipulación material, libre de trabas evidentes que sí condicionaban a la edificación.
Resumen pág 64 | Bibliografía pág 69

Universidad de Navarra. María Villanueva Fernández (Oviedo, 1984), Doctora Arquitecta por la Universidad de Navarra (2012), Premio Luis Moya Fin de Carrera (2007-08). Contratada Doctora por la ANECA (2017). Docente en ETSAUN, TECNUN, ISEM, entre otros centros. Ha difundido su investigación, centrada en el diseño de arquitectos españoles y los límites de esta disciplina con el arte y la arquitectura, en revistas como EGA, PPA, $R A$, entre otras. Estancias de investigación en The Getty Research Institute, LA (2011), en la GSAPP Columbia University, NY (2011) y en ensapbx, Burdeos (2017).

mvillanuevf@unav.es

Universidad de Navarra. Héctor García-Diego Villarías (Santander, 1983), Doctor Arquitecto por la Universidad de Navarra, (2011), Premio Extraordinario Fin de Carrera (2007) y de Doctorado (2013). Docente en la ETSAUN. Contratado Doctor por la ANECA (2016). Subdirector de Ordenación Académica de la ETSAUN (2017). Ha difundido su investigación en revistas como $E G A, P P A, R A$, entre otras. Beca de The Getty Trust para estancia de investigación en The Getty Research Institute de L.A.; beca por la Fundación Bancaja para investigación en la Universidad de Columbia en N.Y. (2011). Estancia de investigación en la ensapbx, Burdeos (2017).

hgarcia-die@unav.es

\section{Palabras clave}

Tecnología, arquitectura, empresas, mobiliario, moderno

Technology, architecture, companies, furniture, modern

Método de financiación Universidad de Navarra
1 El término "Obra de Arte Total" o Gesamtkunstwerk es atribuido al compositor de ópera Richard Wagner quien lo utilizó en 1849 , aunque algunas fuentes indican que fue el escritor y filósofo alemán Trahndorff KFE en un ensayo en 1827 el primero en utilizarlo. Este concepto hace referencia a las obras de 
arte en las que participan varias disciplinas artisticas. En el ámbito de la arquitectura, se utiliza cuando el diseño del arquitecto abarca la totalidad de la obra en todas sus escalas: edificio, mobiliario o elementos decorativos. Aunque la idea de Gesamtkunstwerk no era nueva, está intimamente ligada al estilo Art Nouveau -en todos sus formatos- en el que destacan arquitectos destacados de toda Europa como Josef Hoffmann y Otto Wagner en Austria, Henry van de Velde y Victor Horta en Bélgica, Charles Rennie Mackintosh en Escocia o Antoni Gaudí en España. No obstante, a partir de 1919 la Bauhaus ofrece un nuevo enfoque del concepto, vinculado a la modernidad y de carácter más teórico.

2 Esa transformación radicaba en un cambio no solo decorativo sino integral de la forma, función, material y producción del mueble. La modificación de un estilo a otro variaba principalmente en el aspecto del arte decorativo, en los motivos, líneas que "vestían" al mueble. Sin embargo, en el siglo XX el cambio consistía en algo más profundo que afectaba a todos los aspectos de este. La forma ya no era un producto de la decoración pautada por el estilo sino que respondería, o debería responder, a cuestiones funcionales $y$ ergonómicas.

3 ZABALBEASCOA, Anatxu. "El siglo en sillas". VV.AA. 300\% Spanish Design Barcelona: Sociedad Estatal para Exposiciones Internacionales Barcelona, Sociedad Editorial Electa, 2005. pp. 44-46. ${ }^{4}$ VIDAL, Mercè. "Design history in Catalonia between the influence of Le Corbusier and Mediterranean historical and vernacular sources". VV.AA. Design Discourse, Vol. III, $n^{\circ}$ 4, 2008, p. 4.

5 En 1931, en Zürich fue creada la firma Wohnbedarf, por Giedion, Moser y Graber, realizando exposiciones y creando las primeras colecciones en tubo de acero diseñadas por arquitectos europeos como Werner Max Moser, Flora y Rudolf Steiger, Marcel Breuer, Le Corbusier y Alvar Aalto - web de la firma Wohnbedarf, apartado de historia: https://www.wohnbedarf.ch/de/ ueberuns/geschichte/-. Un año más tarde, en 1932 y 1933, Marcel Michaud realizó varios viajes a Suiza donde entendió el mobiliario de vanguardia europeo como una oportunidad, hasta el punto de lograr un contrato para la importación de piezas de Marcel Breuer o Alvar Aalto y abrir la primera tienda de muebles Stylclair en Lyon. Un año más tarde abrió otra en la misma ciudad con una variedad más amplia de modelos entre los que se encuentran los de Le Corbusier, Charlotte Perriand. OLFSDOTTIR, Asdis. Le mobilier d'Alvar Aalto dans l'espace et dans le temps. La diffusion internationale du design entre 1920-1940. Paris: Publications de la Sorbonne, 2017.

${ }^{6}$ Con respecto al equipamiento del Real Club Naútico de San Sebastián, la revista Arquitectura narraba: "Todos los muebles responden a la idea de comodidad y sentido práctico; en algunas dependencias se han instalado muebles en serie, de la Casa Thonet". LABAYEN, Joaquín; AIZPURUA, José Manuel. "Real Club Náutico de San Sebastián". Arquitectura, n 130,1930 , p. 50. En 1931, la revista A.C. divulgó un texto que presentaba algunos elementos del proyecto;

entre ellos, el mobiliario. Al igual que la revista Arquitectura, A.C. se centró en la elección de piezas de la casa Thonet: "El mobiliario es de la casa Thonet en algunas dependencias, todo él responde a la idea de algo de fácil manejo, limpieza y conservación." "El Club Náutico de San Sebastián", A.C. Documentos de Actividad Contemporánea, n³, 1931, p. 20. Por otro lado, tanto las imágenes publicadas en la revista Arquitectura como las fotografías realizadas por Aizpurua a comienzos de los años 30 y recogidas en el libro José Manuel Aizpurua: Fotógrafo. La Mirada Moderna VV.AA. José Manuel Aizpurua: Fotógrafo. La Mirada Moderna, Catálogo de la Exposición en el Museo Nacional Centro de Arte Reina Sofia, Madrid: Ediciones Aldeasa, 2004permiten ver las sillas tubulares que los arquitectos incluyeron en su Studio.
Esta cuestión sumada, por un lado, a la inexistencia de muebles de calidad y a la ausencia de piezas adecuadas a la nueva arquitectura, por otro, impulsó la labor del arquitecto, quien deseaba poder equipar sus obras con mobiliario acorde a su tiempo y a las necesidades de la sociedad. De este modo, se originó un proceso en el que el mueble alcanzó un protagonismo estelar de la mano de los arquitectos, rompió con la simbología de poder y opulencia que lo caracterizaba y pasó a reflejar, a principios de los años veinte, la modernidad, la vanguardia y el progreso ${ }^{3}$, transformando, a su vez, considerablemente el interior de la arquitectura.

En este proceso fue esencial la colaboración del arquitecto con las empresas de mobiliario; en concreto, con aquellas dispuestas a apostar por el progreso y a innovar tanto en los procesos de producción como en el empleo de nuevos materiales en el sector, dando lugar a formas de mobiliario de vanguardia. El viaje conjunto emprendido por arquitectos y empresas estableció un método de trabajo de aprendizaje y confianza mutuos, y de experimentación técnica y formal, que permitió el desarrollo de nuevos modelos que, de un modo u otro, marcaron la historia del diseño y de la arquitectura. $Y$ que, además, fueron responsables de modificar significativamente su imagen.

\section{Modelos internacionales en la España de entreguerras}

Los nuevos modelos de mobiliario, la innovación material y los pioneros sistemas de producción fueron penetrando en España desde distintos lugares de Europa a través de los catálogos comerciales de las casas de muebles y de las publicaciones, tanto internacionales como nacionales. Entre los casos recogidos en las revistas españolas del periodo de entreguerras, se encuentran los realizados por arquitectos que trabajaron con firmas de muebles con el fin de producir sus diseños surgidos por la necesidad de equipar la nueva arquitectura: Josef Frank y Haus\&Garten- Frank\&Wlach, o Hendrik Sendker y Contempora. Pero, sin duda, las colaboraciones internacionalmente conocidas y difundidas en catálogos y anuncios fueron las de arquitectos como Mart Stam, Mies van der Rohe, Marcel Breuer y Le Corbusier con la casa Thonet, o la de Alvar Aalto con Stylclair y con Artek, fundada por él mismo en 1935.

De hecho, en este proceso de transmisión y desarrollo de las nuevas formas tecnológicas, la colaboración entre arquitectos y empresas de mobiliario españolas adquirió un papel esencial en la realización del diseño moderno en el país durante los años 30. Debido a la popularidad adquirida por este tipo de muebles, aumentó considerablemente la importación de estas piezas producidas en los países del centro de Europa a otras zonas como España. El nuevo mobiliario fue introducido por la firma Thonet, administrado inicialmente por Hermann Heydt a través de sus sucursales en Barcelona, Madrid y Sevilla ${ }^{4}$, tal y como lo muestra la publicidad publicada en la revista A.C. [1] . Por otro lado, Stylclair, creada en 1933 en Lyon por Marcel Michaud, y Wohnbedarf, fundada en 1931 en Zúrich por Sigfried Giedion, Werner Max Moser y Rudolf Graber, comercializaron modelos diseñados por los arquitectos Aalto, Artaria, Breuer, Gropius, Hafeli, Hubacher, Kienzle, Le Corbusier, Merkelbach, Moser, Perriand, Rietveld, Roth, Stam, Steiger, Thomas y Sornay, entre otros ${ }^{5}$.

Algunos de estos nuevos modelos modernos de Thonet y Stylclair fueron distribuidos por MIDVA (Mobles i Decoració de la Vivenda Actual): el espacio expositivo, de reuniones y comercial inaugurado en 1931 por el GATCPAC. Este local, creado con el fin de divulgar el diseño y la arquitectura modernos, se convirtió en el escaparate de la nueva estética moderna procedente de Europa. Pero, además, esta actividad favoreció el desarrollo de una muestra novedosa de productos tecnológicamente avanzados para la nueva arquitectura que se difundiría a través de la revista del grupo y sus miembros. Arquitectos de distintas partes de España, a través del GATCPAC consiguieron piezas de Thonet como, por ejemplo, la silla MR10 de Mies van der Rohe o la silla Wassily de Marcel Breuer, utilizadas por José Manuel Aizpurúa y Joaquín Labayen para equipar el Real Club Náutico de San Sebastián y su estudio respectivamente ${ }^{6}$. [2]

Por tanto, a través de todas estas iniciativas y empresas, podría decirse que en el caso del mobiliario, la tecnología durante la década de los años 30 podía importarse físicamente, lo que facilitó la entrada de manera directa de la nueva estética antes mencionada. Esta difusión tecnológica se produjo no solo a través de casas que importaban modelos originales de arquitectos internacionales realizados con nuevos materiales, sino también mediante firmas de muebles nacionales que llegaron a producir algunas de esas piezas. Es el caso de la firma MAC -Muebles de acero curvado-, fundada por José María Fernández de Castro y Eduardo Shaw Loring, que en 1931 fabricó muebles diseñados por Mies van der Rohe y algunos modelos de Marcel Breuer, no sin verse envuelta en una polémica legal con la firma Thonet ${ }^{7}$. 


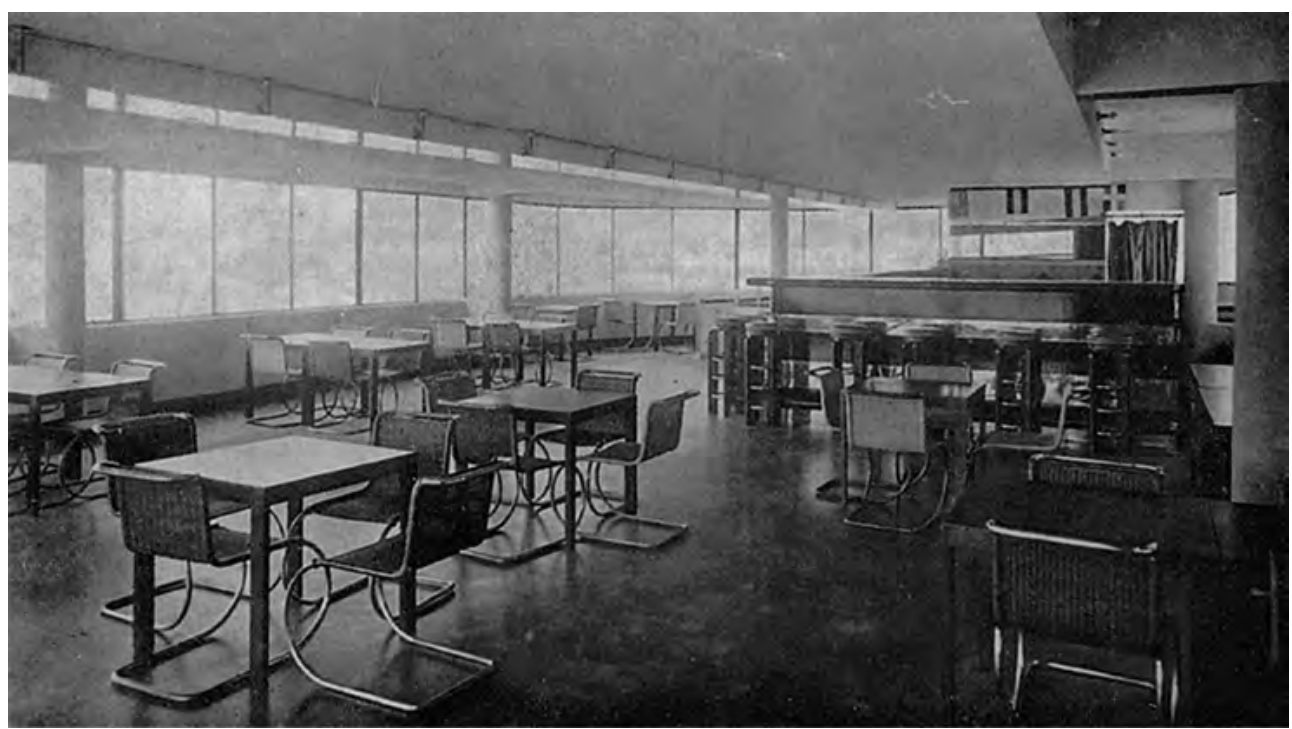

[2]

En Cataluña sucedía algo similar: algunos muebles fueron el producto de la experimentación con técnicas innovadoras en pequeñas empresas como Hermann Heydt, la Vda. J. Ribas o Casa Buades, S.A., en Barcelona ${ }^{8}$. Estas casas aparecieron en las páginas publicitarias, tanto iniciales como finales, de la revista A.C. ${ }^{9}$. Algunas, cobraron especial importancia incluso en los contenidos de la publicación en los que aparecían piezas de mobiliario producidas por ellas y miembros del GATEPAC, como la silla de la Joyería Roca de José Luis Sert ${ }^{10}$ [3]. Otras, tenían presencia en diarios de la época, como la casa Dámaso Azcue o Hermann Heydt. En una de las columnas dedicadas a esta última, el diario $A B C$ (Madrid), exponía que, como "industria alemana en España", destacaba por la importancia que tenía en la arquitectura moderna española, y citaba el nombre de los arquitectos y obras en las que había participado, como signo de modernidad ${ }^{11}$.

A través de la difusión de las firmas y sus productos en los medios escritos y catálogos, los arquitectos no solo se nutrieron de referencias de una nueva estética maquinista, sino que entendieron la empresa como medio para la experimentación propia de nuevas formas y procesos industriales de construcción. Así, las casas de muebles jugaron un papel trascendental en el amueblamiento de proyectos realizados en España, equipándolos con los citados modelos tecnológicos importados y produciendo nuevos prototipos realizados por arquitectos nacionales que emulaban a los extranjeros, tanto en aspectos formales como materiales. De hecho, aquellas empresas comenzaron a colaborar con arquitectos españoles para crear un nuevo mobiliario, heredero de aquella tecnología, pero adaptado a la realidad, por un lado, de la industria española y, por otro, de la arquitectura moderna del país.

\section{Modelos nacionales: el mueble de acero como paradigma}

Ya fuese porque los modelos internacionales no eran fácilmente accesibles, o bien por razones ligadas a la experimentación formal y creativa propia de la disciplina de la arquitectura, un elenco de arquitectos españoles realizó sus propios diseños producidos por empresas nacionales que contribuyeron al desarrollo del mueble moderno y su evolución tecnológica. Aquellos nuevos prototipos emulaban a los extranjeros tanto en su

[3]

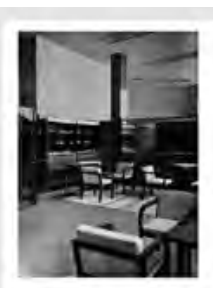

J. RIBAS

MUEBLISTA

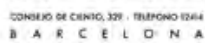

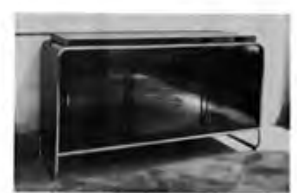

J. RIBAS

MUEBLISTA

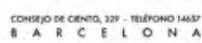

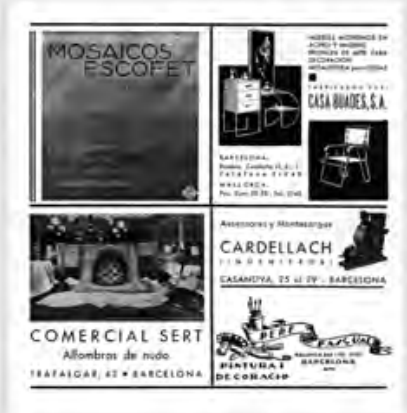

[2] Imagen del interior del Real Club Náutico de San Sebastián equipado con las sillas de Mies van der Rohe. Fuente: Cahiers d'art, $\mathrm{n}^{\circ} 3,1931$

[3] Anuncios de las firmas Vda. J. Ribas y Casa Buades, S.A publicados en la revista A.C. Documentos de Actividad Contemporánea, $\mathrm{n}^{\circ} 14 \mathrm{y} \mathrm{n}^{\circ} 20$.

[4] Portada y página interior del catálogo Stylclair: ses meubles Duralumin, 1934, en el que explica las caracteristicas del nuevo mobiliario: homogénéité, Légèreté y Liberté. 
casa interpreta con justeza el gusto artístico, por muy exquisito que sea, de los facultativos de las obras. Muchos e importantes trabajos ha realizado esta importante casa tanto en edificaciones particulares como oficiales, entre las cuales hacemos mención de las siguientes: Instituto Nacional de Física y Química (Fundación Rockefeller): Arq. Sres. S. Arcas y Lacasa. Edificio de la Junta de la Ciudad Universitaria. Fundación "Del Amo" (Cuidad Universitaria): Arq. Sres. Bergamin y B. Soler. La Unión y El Fénix Español: Arq. Sr. López Otero. Banco Vizcaya en Barcelona: Arq. D. Manuel J. Galindez. Centrales de los Saltos del Alberche: Arq. D. Bellido. Sociedad Española de Fabricación de Automóviles: Arq. Sr. Balbuena. Sociedad Hidroeléctrica Española (Central de Millares) Sociedad Española Naval (San Fernando). Tranvias de Sevilla. Ferrocarriles de San Juan de Aznalfarrache. Instituto - Escuela: Arq. Sr. Arniches." Entre el amplio catálogo de productos que la casa suministraba el anuncio destacaba los muebles de tubo y madera.

12 Entre los arquitectos que amueblaron sus viviendas con alguna pieza de tubo de acero internacional se encuentran Fernando García Mercadal, Rafael Bergamin, Luis Blanco

Soler o Fernando Salvador -"La vivienda del arq. García Mercadal", Viviendas. Revista del Hogar n 8, 1933, pp. 10-15; "La vivienda del arq. R. Bergamín", Viviendas. Revista del $\operatorname{Hogar}^{\circ}$ 8, 1933, pp. 16-20; "La vivienda de arq. L. Blanco Soler", Viviendas. Revista del $\operatorname{Hogar}^{\circ}$ 8, 1933, p. 21; "La vivienda del arq. Fernando Salvador", Viviendas. Revista del Hogar $n^{\circ} 8,1933$, pp. 22-23). Sin embargo, aunque se trataba de excepciones, otros arquitectos como Josep Torres Clavé crearon nuevos modelos de tubo de acero, este los incluyó en su vivienda en Casp92, Barcelona -TORRES, Raimon. et al. Josep Torres Clavé. Barcelona: Santa \& Cole, 1994. p. 18-

${ }^{13}$ No todas las revistas españolas dedicaron el mismo espacio en sus páginas a los interiores realizados por arquitectos españoles y su mobiliario. En la década de los años 20 la revista Arquitectura fue la responsable de mostrar los proyectos de los arquitectos españoles. En el comienzo de los años 30, revistas como A.C., Viviendas, Obras y Nuevas Formas combinaron en mayor medida los contenidos nacionales con los internacionales. Sin embargo, revistas como La Construcción Moderna, La Ciudad Lineal, Cortijos y Rascacielos, Arte y Arquitectura, Arquitectura Española o Archivo Español de Arte y Arquitectura presentaron en sus páginas contenidos predominantemente españoles. Estas revistas mostraron un grupo heterogéneo de arquitectos, si se tiene en cuenta la variedad formal de las piezas de mobiliario que produjeron: desde mobiliario de montaña, como el realizado por José de Yarza, o de tendencias estilisticas del momento y de pasado, como las piezas de Luis Santamaría, hasta muebles vinculados a formas más vanguardistas, en muchos casos similares las extranjeras, como los muebles de José Manuel Aizpurua y Joaquin Labayen.

14 "Exposición de arquitectura y pintura moderna en San Sebastián. Arquitectos: Labayen y Aizpurua. Pastelería y Salón de Degustación Sacha", A.C. Documentos de Actividad Contemporánea, $1^{\circ}$ Trimestre, $\mathrm{n}^{\circ}$

1, 1931; GUTIÉRREZ SOTO, Luis. "El bar Chicote. Nuevo bar en la Gran Via (Madrid)", Arquitectura, $\mathrm{n}^{\circ}$ 150, 1931; GUTIÉRREZ SOTO, Luis. "Bares y cafés", Obras.

Revista de Construcción, n’ 16, 1933; "La Construcción en Madrid. Edificio Carrión (el Capitol)", La Construcción Moderna, n 14 , 1935; "Concurso privado. Solar Carrión en la plaza de Callao", Arquitectura, n 146, 1931; "Arquitectura comercial española. El edificio Carrión de Madrid", Nuevas Formas. Revista de Arquitectura y Decoración, n 1, 1935. "Madrid. El edificio Carrión", Arquitectura, $\mathrm{n}^{\circ}$ 1, 1935.

15 VILLANUEVA FERNÁNDEZ, María, GARCIA-DIEGO, Héctor. "La silla del GATEPAC: un viaje colectivo de ida y vuelta", Proyecto, progreso, arquitectura, $\mathrm{n}^{\circ} 11, \mathrm{pp}$. 43-44. estética como en su material y, de manera menos precisa, en los sistemas de producción. No obstante, esta colaboración e influencia dio lugar a piezas con imagen moderna pero que también reflejaban el carácter de la tradición y la cultura españolas, en parte, debido a la menor capacidad industrial del país.

La asimilación del lenguaje moderno en España se produjo de forma paulatina, tal y como muestran las revistas de arquitectura del periodo. Primero, penetrando en espacios e carácter más público como comercios, cafeterías y bares y, posteriormente, llegando a la vivienda, lugar de mayor intimidad. Curiosamente, este proceso se asemeja al que se produce en el sector del mueble en cuanto al material. Las nuevas formas del extranjero penetran en las obras dedicadas al ocio a través de modelos realizados con materiales como el acero, mientras que en la vivienda predomina el uso de la madera y el mimbre, que vinculan las nuevas formas con la modernidad y la tradición españolas. No obstante, en algunas viviendas, especialmente las de algunos arquitectos, fue más habitual la inclusión de piezas de acero, en concreto las diseñadas por sus homólogos europeos; de manera que la experimentación formal y creación de modelos propios se desarrolla principalmente en el campo de la arquitectura del ocio ${ }^{12}$.

De en torno al casi medio centenar de arquitectos españoles que aparecieron en las revistas de arquitectura nacionales por diseñar mobiliario ${ }^{13}$, tan solo unos pocos protagonizaron artículos en los que se difundía la colaboración arquitecto-empresa y aún en menos destacaban por su actitud experimental y moderna, tanto en la forma como en el empleo de nuevos materiales. Aunque, en realidad, el grupo fue más amplio, las piezas de arquitectos españoles, producidas en tubo de acero y publicadas en las revistas fueron escasas, destacando por su difusión e innovación, las que equiparon los interiores de la Pastelería Sacha de José Manuel Aizpurúa y Joaquín Labayen, del Bar Chicote y del Café Aquarium de Luis Gutiérrez Soto, y del Edificio Capitol de Luis M. Feduchi ${ }^{14}$ Estos proyectos de mobiliario, planteados con gran ambición, fueron posibles gracias al trabajo y al apoyo técnico de las empresas Rolaco-Mac y Dámaso Azcue que permitieron la cristalización de las formas modernas en reinterpretaciones de las piezas icónicas internacionales, gracias a su postura experimental en el empleo de nuevos materiales como el tubo de acero.

Este material que ofrecía grandes y novedosas posibilidades de manipulación y liviandad, permitía, debido a la resistencia del material, disminuir la sección estructural, en algunos casos incluso simulando desafiar las leyes de la física y proporcionando mayor "libertad" en el movimiento del usuario, como anunciaba la casa Stylclair [4]. Por ello, los arquitectos potencialmente modernos sentían cierta predilección por el tubo de acero, como así sucedió con José Manuel Aizpurua y Joaquín Labayen, quienes lo introdujeron en su arquitectura -al inicio a través de modelos existentes, como el caso citado anteriormente del Real Club Náutico de San Sebastián equipado con piezas de Thonet, y, poco después, con sus propios diseños que eran variaciones de los primeros. Prueba de ello son los dibujos de sillas encontrados en el Archivo Labayen que comparten un asombroso parecido con modelos de la casa Thonet como la silla Cesca de Marcel Breuer ${ }^{15}$ [5]. Mediante la inspiración de modelos internacionales realizados en tubo de acero, estos arquitectos se familiarizaron con aquellas formas venidas de Europa y propusieron nuevos modelos, como la silla diseñada para la Pastelería y Salón de Degustación Sacha de San Sebastián (1930).

Aizpurua y Labayen entendian la elección del equipamiento como un aspecto medular en el proyecto de arquitectura, algo que queda patente en sus planos y perspectivas [6]. Además,

[4]
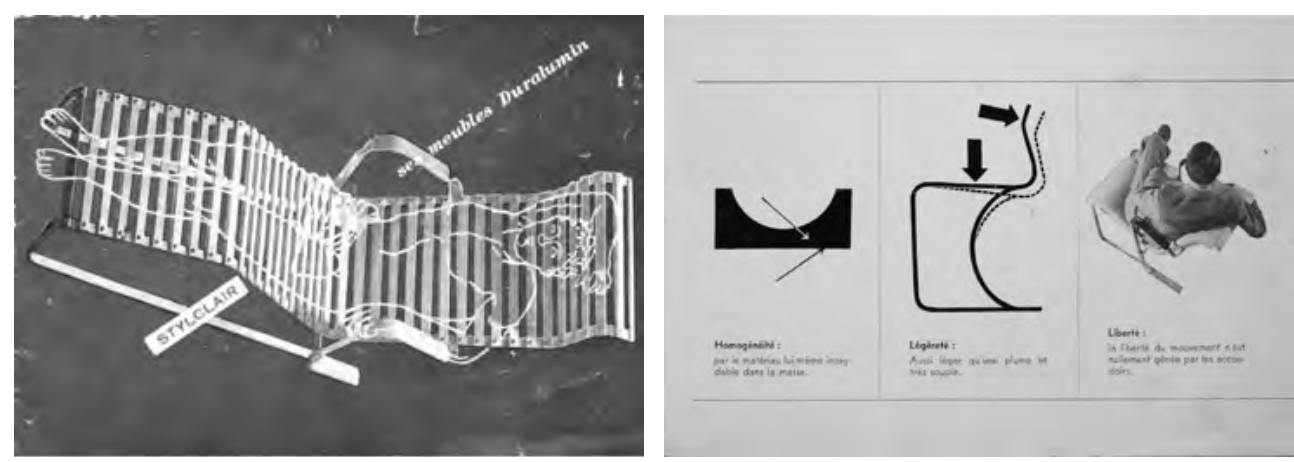
nombrado director artístico. Con el crecimiento de la empresa se construyeron una nueva fábrica y oficinas, siendo la primera incautada en 1936 por el Ministerio de la Guerra para fabricar espoletas. Los muebles que se encontraban alli fueron vendidos o requisados para las oficinas de los ministerios. Durante la Guerra Civil, la empresa permaneció inactiva y su fábrica cerrada. Tras el conflicto, la firma reabre sus puertas con Goicoechea como director artistico y es en 1944 cuando Luis M. Feduchi se convierte en director artístico de la empresa. BLASCO CASTIÑEIRA, Selina. Biografía de Luis Feduchi (Archivo Ignacio Feduchi); BLASCO CASTIÑEIRA, Selina. Luis Feduchi 1901-1975, Monografias de arquitectos españoles. Dirección General de Arquitectura, MOPU, p. 33; FEDUCHI, Luis. "Breve historia de Rolaco", Experimenta: Diseño del mueble $n$ España 1902-1998 (Madrid), n 20, 1998, p. 20. Conversación con Ignacio Feduchi, 19 de diciembre de 2011.

${ }^{25}$ FEDUCHI, Luis. "Breve historia de Rolaco", Experimenta: Diseño del mueble en España 1902-1998 (Madrid), nº 20, 1998, p. 20.

${ }^{\mathbf{2 6}}$ BLASCO CASTIÑEIRA, Selina. "Pesquisas, recuerdos y una entrevista", Feduchi: tres generaciones en arquitectura $y$ diseño, Tabernes Blanques (Valencia): Malabar Serveis Grafics i Edit, 2009, p. 12.

${ }^{27}$ VILLANUEVA FERNÁNDEZ, María, GARCIAA-DIEGO Héctor. "Una aventura empresarial en un proyecto integral: Mobiliario del edificio Capitol, Luis M. Feduchi, 1931331" Res Mobilis. Revista internacional de investigación en mobiliario y objetos decorativos. Vol. 6, $n^{\circ} 7,2017$.

$\mathbf{2 8}^{28}$ FULLAONDO, Juan Daniel. Los muebles del Capitol. Madrid: B.D. Ediciones de Diseño, 1980, p. 59; VV.AA El diseño industrial en España, Madrid: Cátedra, 2010, p. 83; FERNÁNDEZ SINDE, Alfredo. Luis M. Feduchi Arquitecto y diseñador: una investigación sobre el edificio Carrión, Tesis Doctoral. Mayo, 1995

${ }^{29}$ Gutiérrez Soto realizó varios proyectos de arquitectura para el ocio en la década de los años 30: el café Aquarium, el bar Maria Cristina o el dancing salón de té Casablanca, pero el más conocido de todos ellos fue el Bar Chicote, no solo por la intervención del arquitecto sino también por tratarse de uno de los locales más emblemáticos de Madrid en aquella época y sucesivas. Situado en la Gran Via y fundado por Perico Chicote, era conocido por sus cócteles, atrayendo a personajes de fama internacional de todos los ámbitos. Rainiero de Mónaco, Ernest Hemingway, Frank Sinatra, Ava Gardner, Rita Hayworth o Sofia Loren, entre otros.

${ }^{30}$ GUTIÉRREZ SOTO, Luis. "Bares y cafés", Obras. Revista de Construcción, n ${ }^{\circ} 16,1933$. pp. 60-68.

${ }^{31}$ Estas piezas fueron combinadas con un elemento continuo de asiento incorporado en un gran zócalo que recorría todo el local adherido a los paramentos como parte de la propia arquitectura. En la zona más exterior cualquier intervención posterior" ${ }^{25}$, dando "empuje y visibilidad a la empresa" ${ }^{26}$ en el mundo del mueble y la arquitectura modernos.

En el proyecto de mobiliario desarrollado por Luis M. Feduchi también intervinieron otras firmas embarcándose en una verdadera aventura empresarial ${ }^{27}$. La heterogeneidad funcional del edificio -que contenía uso residencial, cafeterías, restaurantes y cines, entre otros- requería mobiliario adecuado a cada ambiente. Esta variedad de modelos demandaba también una diversidad de materiales, definiendo también la elección de la empresa de mobiliario. Feduchi empleó madera maciza para las piezas más conservadoras o de estilo realizadas por Santamaría y Crowner, y madera y acero curvados para piezas más modernas llevadas a cabo por Lledó y Rolaco-Mac, destacando esta última por el empleo del tubo de acero en varias piezas ${ }^{28}$.

Aunque fueron varias las piezas realizadas con este material en este proyecto, como, por ejemplo, el conocido carrito-bar producido por Rolaco junto con la empresa Lledó y diseñado siguiendo una geometría curva similar a la de la fachada del propio edificio, o las butacas para la sala de cine, similares a las del Cine Actualidades, destaca por su vinculación formal con modelos internacionales y por su innovación técnica especialmente la silla para la terraza de la Gran Vía. Este modelo, el más desconocido, recordaba a otros ejemplos europeos modernos como las sillas cantiléver de Mies o Breuer. Su estructura era de tubo de acero curvado con asiento y respaldo de médula. El empleo de estos materiales favorecía el desarrollo de geometrías curvas, dibujando en la base un juego serpenteante, como los que realizaban Jean Burkhalter o Erich Dieckmann, que proporcionaba un carácter original a esta tipología de silla [10].

Otro arquitecto que colaboró con la casa Rolaco fue Luis Gutiérrez Soto para el proyecto del emblemático Bar Chicote ${ }^{29}$. En esta obra el arquitecto proyectó un espacio con dos ambientes diferenciados ${ }^{30}$, aspecto que determinaría a su vez el proyecto de diseño del mobiliario. Mientras la zona más alejada de la calle tenía un carácter más lineal, con la barra a un lado y unas cabinas al otro ${ }^{31}$; la otra, de proporciones más regulares, hacía de vestíbulo del bar pero también de escaparate y llamada de atención. Gutiérrez Soto empleó dos lenguajes distintos en el proyecto de mobiliario con el objetivo de delimitar visualmente estos ambientes que estaban determinados por el tipo de experiencia y los grados de privacidad que el usuario quería tener. El espacio interior, más conservador, proporcionaba una mayor intimidad a los usuarios, a diferencia de la zona más exterior que exponía a los clientes hacia la calle tras un gran vidrio situado en fachada que hacía de gran escaparate ${ }^{\mathbf{3 2}}$,

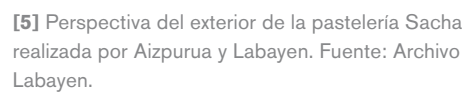
Labayen.

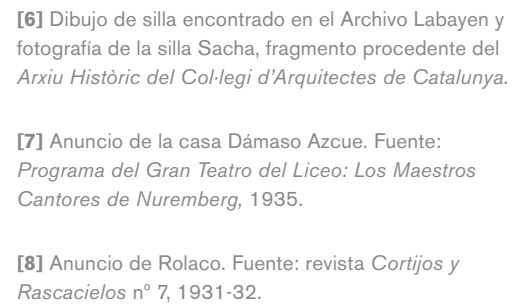

Rascacielos $n^{\circ}$ 7, 1931-32.

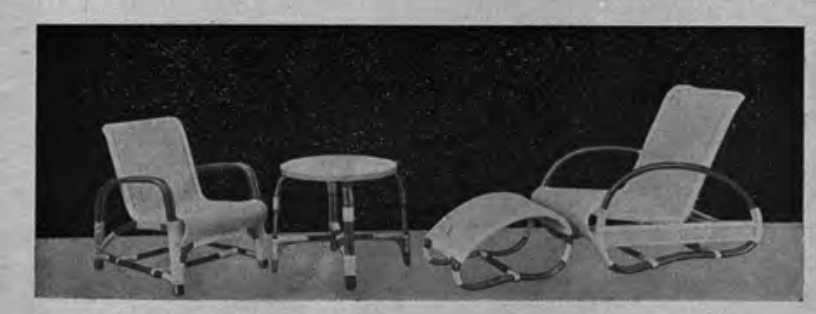

Creaciones en muebles modernos de médula combinada con malaca

\section{Casa Dámaso Azcue}

FABRICA EN AZPEITIA SUCURSAL EN BARCEL ONA,
RAMBLA DE LAS FLORES, IS

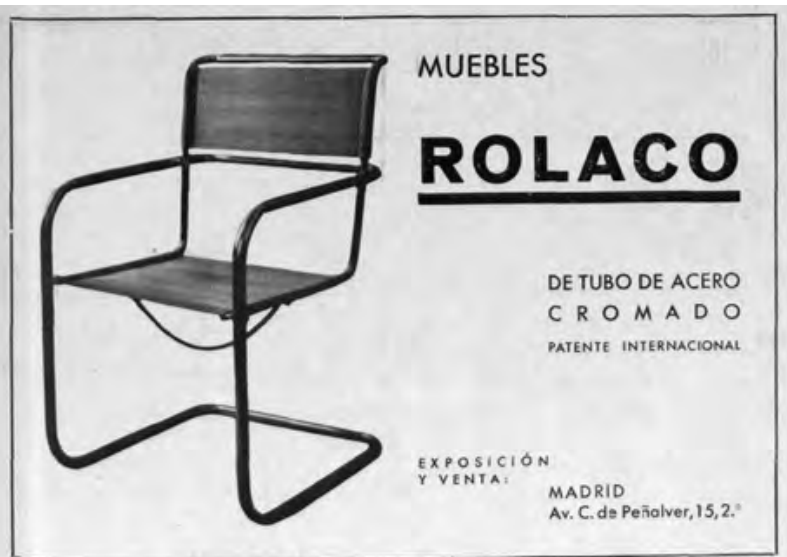


un aspecto frecuente en los nuevos locales de ocio, heredado de la modernidad e inspirado en los locales comerciales. [11]

En concreto, en esta última parte, aprovechando la exposición directa a la Gran Vía, Gutiérrez Soto diseñó una silla construida con estructura de tubo de acero cromado y con respaldo y asiento tapizados en tela [12]. El modelo guardaba cierta similitud con una de las sillas diseñadas por Eileen Gray en 1929, aunque la estructura describía una sección parecida, la pieza del arquitecto español se reforzaba con un rigidizador y el respaldo se encajaba en la estructura sin llegar a sobresalir. Este modelo, realizado con nuevos materiales e innovadores sistemas de producción por la casa Rolaco, posibilitó su seriación, equipando otras obras de la época como el café Aquarium ${ }^{33}$, también de Gutiérrez Soto.

\section{El mobiliario como vehículo de modernización de la arquitectura}

Tras esta aproximación es posible extraer algunas reflexiones acerca de las relaciones establecidas en el periodo de entreguerras entre las empresas de mobiliario y los arquitectos españoles modernos. Como se ha podido ver, una primera apreciación es que la colaboración entre ambos se produjo de manera bidireccional. Más allá de entenderla como una relación con intereses paralelos o dispares, en la que las empresas proporcionaron los medios y los arquitectos aportaron el conocimiento de interpretación de una época, una sociedad y un contexto, parece tratarse en mayor medida de una operación conjunta con objetivos compartidos. Por un lado, los arquitectos pudieron ver materializada su creatividad y equipar su arquitectura con piezas adecuadas a ella, al tiempo que experimentaron con las nuevas formas y materiales, en modelos reales. Por otro, la empresa aprovechó las ideas de los arquitectos para innovar y progresar tecnológicamente pero, también, como estrategia de mercado para distinguirse de otras casas de muebles y posicionarse en lo alto del sector del mobiliario vanguardista. En ambos casos la colaboración se entendió como laboratorio para el desarrollo de la imagen de modernidad.

Otra reflexión que queda patente es que la ideación de nuevos tipos permitió a ambas partes experimentar con las nuevas formas e innovar con los procesos tecnológicos, destacando especialmente el empleo de nuevos materiales como el tubo de acero. Este material ofreció grandes posibilidades formales -disminución de la sección, nuevas morfologías estructurales, una aparente sensación de liviandad y mayor libertad de movimiento en el usuario-, convirtiéndolo en imagen y signo de modernidad no solo en el extranjero, sino también en España. Aquellos modelos, inicialmente producidos de forma artesanal, alcanzaron tecnologías que permitieron industrializar el proceso de producción. Este desarrollo favoreció la seriación, más o menos industrializada, de piezas y constituyó una oportunidad no solo en el avance tecnológico sino también en el formal del propio mueble que trascendió al plano de la arquitectura.

El mobiliario se convirtió entonces en una herramienta eficaz de modernización del espacio. En ocasiones, los arquitectos españoles no podían proyectar una arquitectura alineada a las ideas racionalistas o, por el contrario, querian reforzarla, y en ambos casos muchos de ellos empleaban el mobiliario moderno para crear o potenciar una imagen vanguardista del espacio. Algunos arquitectos amueblarían sus obras con los modelos internacionales de arquitectos como Mies van der Rohe o Marcel Breuer producidos por empresas como Thonet, Stylclair o incluso españolas como MAC; otros, sin embargo, crearon nuevos tipos junto con empresas como Rolaco o Dámaso Azcue, inspirados en los modelos internacionales que veían en los catálogos y revistas.

Gran parte de estas colaboraciones se producen en proyectos en los que su uso o propiedad están vinculados de algún modo a la modernidad, como por ejemplo los nuevos espacios de ocio o para la educación. Esta modernidad, más conceptual, que caracterizaba al espacio arquitectónico se vio reforzada con piezas de mobiliario modernas, adecuadas no solo a la nueva arquitectura sino a la vanguardia del proyecto generador. Esta estrategia lograba dotar de una imagen moderna a los espacios de aquella época, a través del mobiliario, entendiendo el proyecto como arquitectura equipada y no como dos elementos independientes. Por esta razón, la elección de la empresa y su producto era cardinal, como se aprecia en los proyectos de Aizpurua y Labayen para la Pastelería Sacha, de Feduchi para el edificio Capitol o de Gutiérrez Soto para el Bar Chicote. En ellos el mobiliario no solo equipó la arquitectura sino que también formó parte del proyecto arquitectónico diferenciando los distintos espacios y trasladando al exterior una imagen de progreso y modernidad.

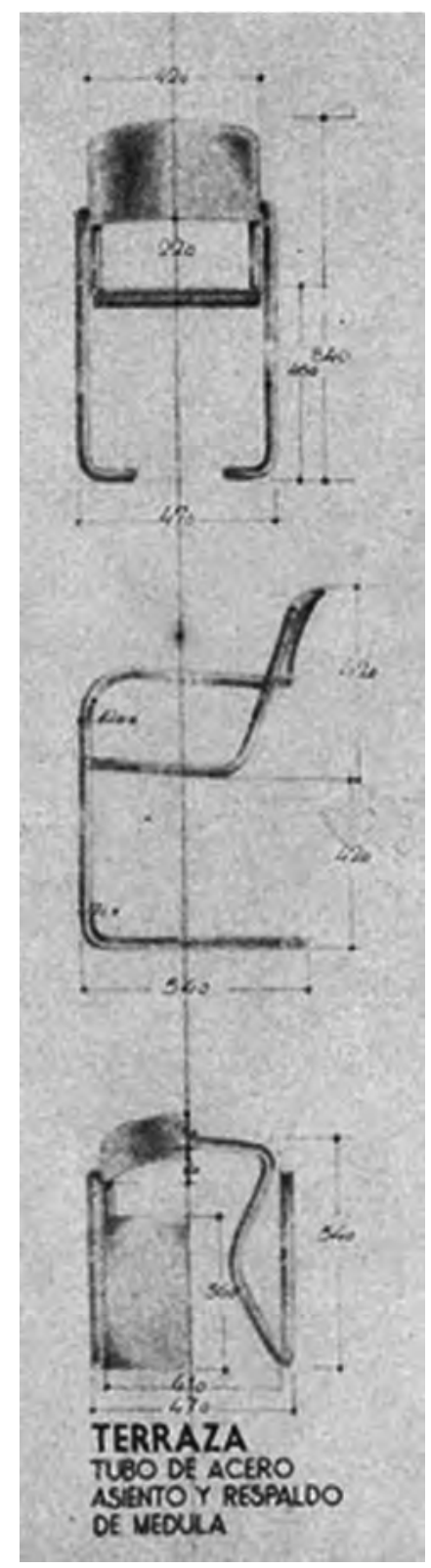

[9]

este elemento se convertía en banco corrido mientras que en la interior hacía de barra, cabinas y elemento de separación.

32 GUTIÉRREZ SOTO, Luis. "Bares y cafés", Obras. Revista de Construcción, n¹6, 1933 p. 68. GUTIÉRREZ SOTO, Luis. "El bar Chicote. Nuevo bar en la Gran Via (Madrid)", Arquitectura, n¹50, 1931, pp. 351-356.

33 "Reforma del café Aquarium en Madrid", Nuevas Formas. Revista de arquitectura y decoración, nº10, 1934, pp. 505-517. 
[9] Planos de la silla para la terraza del edificio Capitol dibujado por el arquitecto Luis M. Feduchi. Fuente: revista Arquitectura, $n^{\circ}$ 1, Madrid, 1935; Documento original en Archivo I. Feduchi.

[10] Fotografias interiores del Bar Chicote de Luis Gutiérrez Soto. Fuente: revista Arquitectura, $\mathrm{n}^{\circ} 150,1931$

[11] Sillas Chicote en el espacio de

entrada, diseñadas por Luis Gutiérrez Soto y producidas por Rolaco. Fuente: Arquitectura, $n^{\circ} 150,1931$.
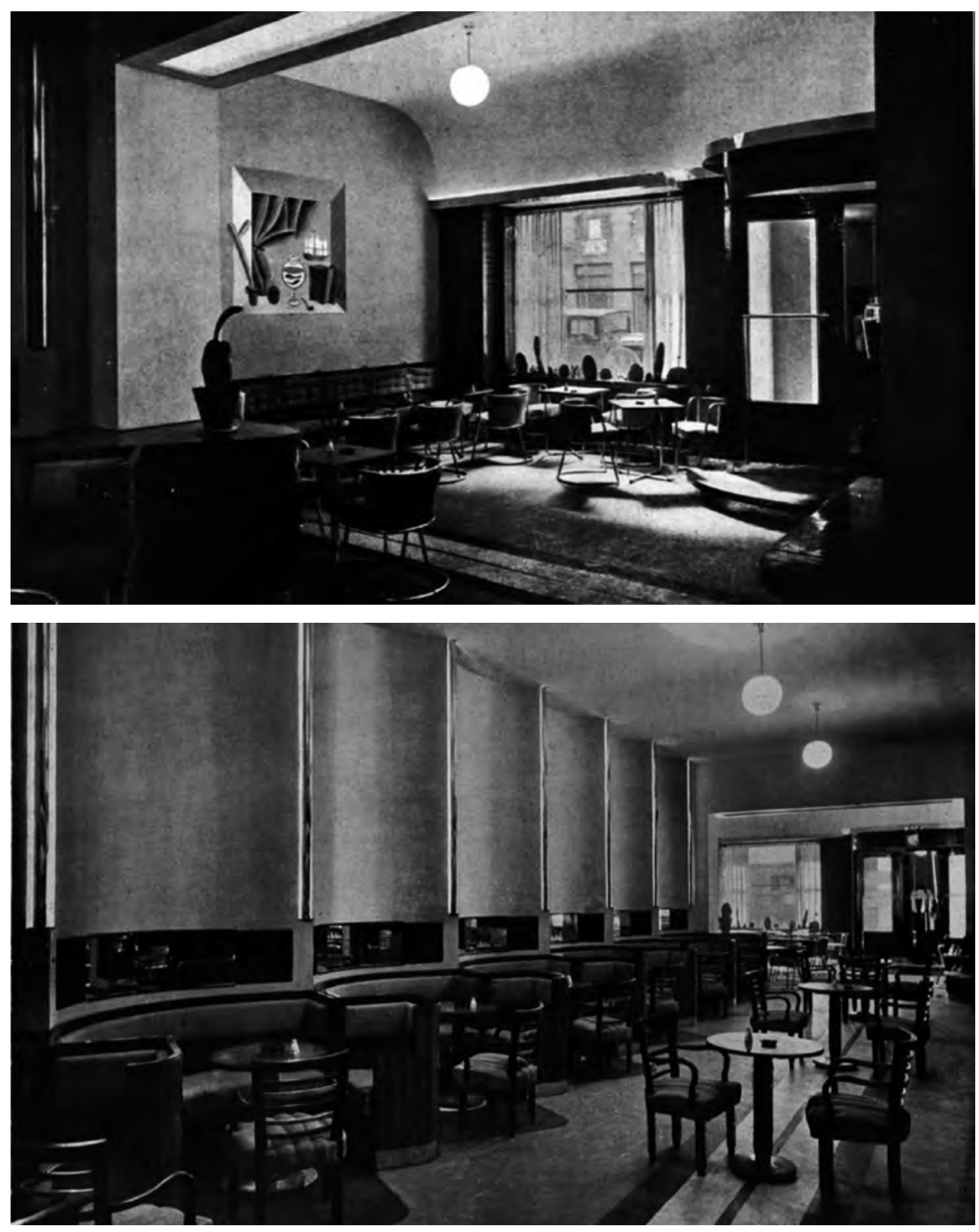

[10]

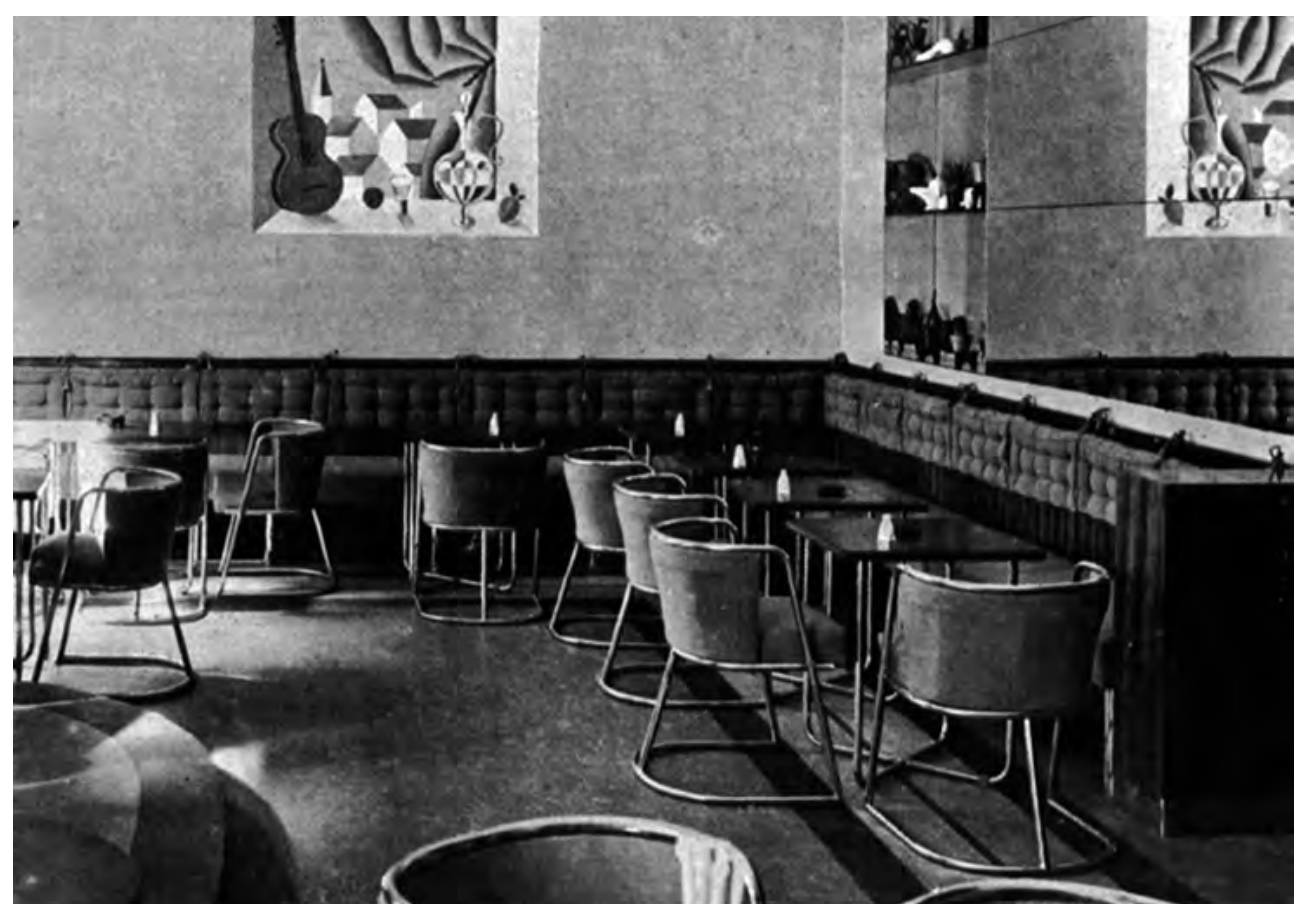

[11] 


\section{3 | Tecnología posible. El mobiliario como vehículo de modernización de la arquitectura española de los años} $30 \_$María Villanueva Fernández; Héctor García-Diego Villarías

Después de la I Guerra Mundial, se produjo un progreso significativo en el sector tecnológico que se convirtió en el fundamento caracterizador de la modernidad en la arquitectura, no solo por la experimentación material y de producción, sino también por la creación de una estética maquinista. Mientras que las innovaciones tecnológicas penetraban gradualmente en la arquitectura, el mobiliario permitía el ensayo de las nuevas técnicas de manipulación material. En este proceso, la colaboración entre arquitectos y empresas de mobiliario españolas adquirió un papel cardinal en el diseño moderno en el país durante los años 30 . A través del análisis de varios casos de estudio, esta investigación evidencia la influencia del trabajo de los arquitectos extranjeros en el diseño de mobiliario español y el papel de las empresas, en colaboración con los arquitectos españoles, como catalizadoras de las nuevas corrientes. Con ello se persigue un único objetivo: mostrar el mobiliario como ejercicio de experimentación tecnológica y vehículo de modernización de la arquitectura.

\section{Palabras clave}

Tecnología, arquitectura, empresas, mobiliario, moderno
03 | Possible technology. The furniture as a vehicle for the modernization of Spanish architecture in the $30^{\mathrm{s}}$ _María Villanueva Fernández; Héctor García-Diego Villarías

After the I World War, technological sector experienced a significant progress, the background of modernity in architecture had been improved, not only by the experimentation of material and production, but also by the creation of a machinist aesthetic. Meanwhile technological innovations gradually spread across architecture the material allowed the testing of new material handling techniques. In this process, the collaboration between Spanish architects and furniture companies acquired a cardinal role in modern design in the country during the $30^{\mathrm{s}}$. Through the analysis of several case studies and providing original documentation, this research shows the influence of the work of foreign architects on the design of Spanish furniture in the $30^{\mathrm{s}}$ and the role of companies, in collaboration with Spanish architects, as catalysts of the new avant-garde currents. With this, a single objective is pursued, which is to show the furniture as an exercise in technological experimentation and a vehicle for the modernization of architecture.

Keywords

Technology, architecture, companies, furniture, modern

\section{3 | Tecnología posible. El mobiliario como vehículo de modernización de la arquitectura española de los años}

$30 \_$María Villanueva Fernández; Héctor García-Diego Villarías

"Arquitectura comercial española. El edificio Carrión de Madrid", Nuevas Formas. Revista de Arquitectura y Decoración, n¹, 1935.

"Concurso privado. Solar Carrión en la plaza de Callao", Arquitectura, n¹46, 1931.

"El Club Náutico de San Sebastián", A.C. Documentos de Actividad Contemporánea, n³, 1931.

"Exposición de arquitectura y pintura moderna en San Sebastián. Arquitectos: Labayen y Aizpurua. Pastelería y Salón de Degustación Sacha", A.C.

Documentos de Actividad Contemporánea, $1^{\circ}$ Trimestre, n¹, 1931, pp. 18-19.

"La Construcción en Madrid. Edificio Carrión (el Capitol)", La Construcción Moderna, n¹4, 1935.

"La vivienda del arq. Fernando Salvador", Viviendas. Revista del Hogar n 8, 1933.

"La vivienda del arq. García Mercadal", Viviendas. Revista del Hogar n 8, 1933.

"La vivienda del arq. L. Blanco Soler", Viviendas. Revista del Hogar n 8, 1933.

"La vivienda del arq. R. Bergamín", Viviendas. Revista del Hogar n 8, 1933.

"Madrid. El edificio Carrión", Arquitectura, n¹, 1935.

"Reforma del café Aquarium en Madrid", Nuevas Formas. Revista de arquitectura y decoración, n¹0, 1934.

AZCUE, Dámaso. Anuncio publicitario, Programa del Gran Teatro del Liceo: Los Maestros Cantores de Nuremberg, 24-1-1935.

BLASCO CASTIÑEIRA, Selina. Biografía de Luis Feduchi (Archivo Ignacio Feduchi).

BLASCO CASTIÑEIRA, Selina. Luis Feduchi 1901-1975, Monografías de arquitectos españoles. Dirección General de Arquitectura, MOPU.

BLASCO CASTIÑEIRA, Selina. "Pesquisas, recuerdos y una entrevista", Feduchi: tres generaciones en arquitectura y diseño, Tabernes Blanques (Valencia): Malabar Serveis Grafics i Edit, 2009.

CAPELLA, Juli; LARREA, Quim. Nuevo diseño español. Barcelona: Gustavo Gili, 1991.

FEDUCHI, Ignacio. Conversación mantenida 19 de diciembre de 2011.

FEDUCHI, Luis. "Breve historia de Rolaco". Experimenta: Diseño del mueble en España 1902-1998 (Madrid), n²0, 1998.

FERNÁNDEZ SINDE, Alfredo. Luis M. Feduchi Arquitecto y diseñador: una investigación sobre el edificio Carrión, Tesis Doctoral. Mayo, 1995.

FULLAONDO, Juan Daniel. Los Muebles del Capitol. Madrid: B.D. Ediciones de Diseño, 1980.

GATEPAC. "Joyería Roca, Barcelona", A.C. Documentos de Actividad Contemporánea, 2 Trimestre, n¹4, 1934.

GUTIÉRREZ SOTO, Luis. "Bares y cafés", Obras. Revista de Construcción, n¹6, 1933.

GUTIÉRREZ SOTO, Luis. "El bar Chicote. Nuevo bar en la Gran Vía (Madrid)", Arquitectura, n¹50, 1931.

HEYDT, Hermann. Anuncio publicitario, Abc (07/08/1934).

JIMENEZ DE LA NAVA, María. "Rolaco. Estudio histórico de la marca". Trabajo Fin de Grado. Tutor: Pedro Feduchi. Departamento de Proyectos

Arquitectónicos. Escuela Técnica Superior de Madrid, Universidad Politécnica de Madrid, junio 2017.

LABAYEN, Joaquín; AIZPURUA, José Manuel. "Real Club Náutico de San Sebastián”, Arquitectura, n¹30, 1930.

OLFSDOTTIR, Asdis. Le mobilier d'Alvar Aalto dans l'espace et dans le temps. La diffusion internationale du design entre 1920-1940. París: Publications de la Sorbonne, 2017.

SAN VICENTE, Jesús. "Entrevista a Javier Feduchi", Experimenta: Diseño del mueble $n$ España 1902-1998 (Madrid), n²0, 1998.

TORRES, Raimon. et al. Josep Torres Clavé. Barcelona: Santa \& Cole, 1994.

VV. AA. El diseño industrial en España, Madrid: Cátedra, 2010.

VV. AA. José Manuel Aizpurua: Fotógrafo. La Mirada Moderna, Catálogo de la Exposición en el Museo Nacional Centro de Arte Reina Sofía, Madrid:

Ediciones Aldeasa, 2004.

VIDAL, Mercè. "Design history in Catalonia between the influence of Le Corbusier and Mediterranean historical and vernacular sources", Design Discourse, Vol. III $n^{\circ} 4,2008$.

VILLANUEVA FERNÁNDEZ, Maria; GARCÍA-DIEGO, Héctor. "La silla del GATEPAC: un viaje colectivo de ida y vuelta", Proyecto, progreso, arquitectura $\mathrm{n}^{\circ} 11$.

WOHNBEDARF web: https://www.wohnbedarf.ch/de/ueberuns/geschichte/

ZABALBEASCOA, Anatxu. "El siglo en sillas", VV.AA. 300\% Spanish Design. Barcelona: Sociedad Estatal para Exposiciones Internacionales Barcelona,

Sociedad Editorial Electa, 2005 